\title{
Postoperative Gastrointestinal Hemorrhage in Biliary Atresia
}

\author{
Tsuneo Chiba, Izumi Mochizuki and Ryoji OHI \\ Department of Pediatric Surgery, Tohoku University School \\ of Medicine, Sendai 980
}

\begin{abstract}
Снiва, Т., Мосніzuкi, I. and Онг, R. Postoperative Gastrointestinal Hemorrhage in Biliary Atresia. Tohoku J. Exp. Med., 1990, 162 (3), 255-259 During the past 9 years, we have treated 23 patients with gastrointestinal hemorrhage following corrective surgery for biliary atresia. Ulcers or erosions of the stomach, duodenum or intestinal wall were observed endoscopically in 20 cases. In other 3 cases, bleeding point was not determined endoscopically, but massive bleeding was recognized frequently. Ten patients bled within 6 months of surgery, and the incidence of bleeding was also seen even after the age of 10 years. A history of cholangitis, the presence of jaundice and the use of cholagogues were related to the postoperative gastrointestinal bleeding. Serum concentrations of gastrin and gastric inhibitory polypeptide showed high values after surgery in almost half the biliary atresia patients examined, however, no clear relationship between the concentration of these factors and gastrointestinal hemorrhage was established.—— biliary atresia; gastrointestinal hemorrhage; peptic ulcer; gastrin; gastric inhibitory polypeptide
\end{abstract}

Many postoperative complications have been reported following corrective surgery for biliary atresia (Altman et al. 1975 ; Odievre 1980), and severe gastrointestinal hemorrhage may be fatal (Saeki et al. 1987). The detection rate of ulcers or erosions has increased with the increased use of endoscopy. We have treated 23 patients for postoperative gastrointestinal hemorrhage associated with biliary atresia during the past 9 years. The clinical factors related to gastrointestinal hemorrhage in these patients are investigated in this study.

\section{Materials And Methods}

From January 1981 to December 1989, 23 patients, 7 males and 16 females, were admitted for gastrointestinal hemorrhage, or developed hemorrhage during admission for biliary atresia. All patients except 3 were determined endoscopically to have gastrointestinal ulcers or erosions. Clinical background and the endoscopic findings in these 23 patients were reviewed. Furthermore, to investigate the etiology of this condition, 14 patients with biliary atresia underwent measurement of pre- and postoperative serum gastrin and gastric inhibitory polypeptide (GIP) concentrations. Serum gastrin concentration was determined by radioimmunoassay (RIA), with normal defined as less than $100 \mathrm{pg} / \mathrm{ml}$. The GIP concentration also was determined by RIA, with normal defined as less than $200 \mathrm{pg} / \mathrm{ml}$.

Received October 3, 1990; revision accepted for publication October 27, 1990. 


\section{Results}

The clinical profile of the 23 patients is summarized in Table 1. There were no differences in the age at operation or portal vein pressure between these patients and non-bleeding patients. Seventeen patients, including 6 of 7 males, had a history of cholangitis. Esophageal varices were detected in only 4 patients at the time the bleeding occurred, and two third of the patients had jaundice.

Peptic ulcers occurring after surgery for biliary atresia were presented as

TABLE 1. Clinical profile of patients

\begin{tabular}{lccc}
\hline & Male & Female & Total \\
\hline Number of patients & 7 & 16 & 23 \\
Age at operation (days) & & & \\
-60 & 1 & 5 & 6 \\
$61-90$ & 4 & 7 & 11 \\
$91-$ & 2 & 4 & 6 \\
Protal vein pressure (-mmH $\left.{ }_{2} \mathrm{O}\right)$ & & & \\
-200 & 1 & 4 & 5 \\
201-300 & 2 & 8 & 10 \\
$301-$ & 1 & 1 & 2 \\
Unknown & 3 & 3 & 6 \\
History of cholangitis & & & \\
Yes & 6 & 11 & 17 \\
No & 1 & 5 & 6 \\
Esophageal varices & & & \\
Yes & 3 & 1 & 4 \\
No & 4 & 15 & 19 \\
Yes & & & \\
No & 3 & 11 & 14 \\
Jaundice & 4 & 5 & 9 \\
\hline
\end{tabular}

TABLE 2. Interval between initial operation and gastrointestinal hemorrhage

\begin{tabular}{rccc}
\hline & Male & Female & Total \\
\hline Within 6 months & 1 & 9 & 10 \\
- 1 year & 2 & 2 & 4 \\
- 2 years & 0 & 3 & 3 \\
- 5 years & 0 & 0 & 0 \\
-10 years & 0 & 0 & 0 \\
More than 10 years & 4 & 2 & 6 \\
\hline
\end{tabular}


gastrointestinal hemorrhage. Table 2 shows the intervals between the initial operation and bleeding. The largest number of patients, 10, had bleeding within 6 months after the operation. Thereafter the incidence of bleeding gradually decreased bleeding after the age of 10 years, including 2 adults. The most common site of bleeding was the duodenum (9 patients), followed by stomach (4 patients) and other sites. Only 2 patients had bleeding from both the stomach and duodenum. In 3 cases, however, the site of bleeding was not determined endoscopically. Massive bleeding was recognized in these patients. Bleeding from the intestinal wall was suspected.

Drugs appeared to be involved in the development of ulcers and erosions. Steroids were used in all patients, and glucagon and prostaglandin $\mathrm{E}_{2}$ were used in one third of the patients. In about half the patients, the bleeding occurred during or immediately after the administration of these drugs.

The serum gastrin concentration was measured in 14 patients, including 4 with bleeding, to study the factor of gastrointestinal ulcers following surgery for biliary atresia (Fig. 1). The number of patients with a concentration greater than $100 \mathrm{pg} / \mathrm{ml}$ increased postoperatively. A similar trend was observed in the serum GIP level (Fig. 2). However, gastrointestinal hemorrhage was uncommon even when these concentrations were abnormally high. Furthermore, there was no relationship between these concentrations and the existence of jaundice.

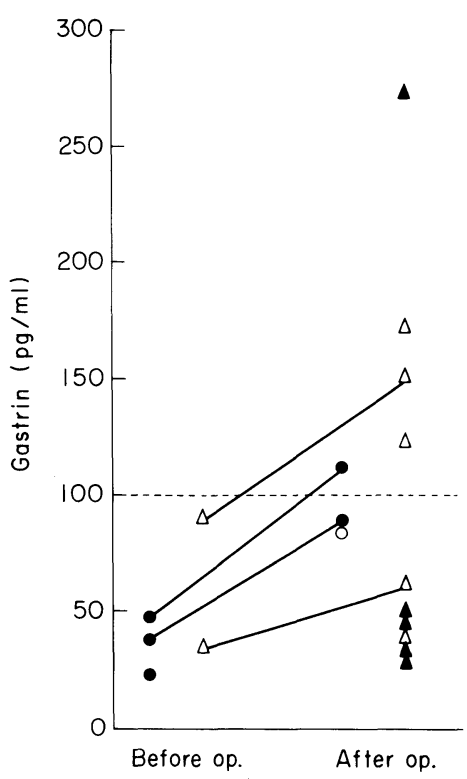

Fig. 1. Serum gastrin level in biliary atresia patients. $\bigcirc$, bleeding and jaundicefree; $\bullet$, bleeding and jaundiced ; $\triangle$, nonbleeding and jaundice-free ; $\Delta$, nonbleeding and jaundiced. 


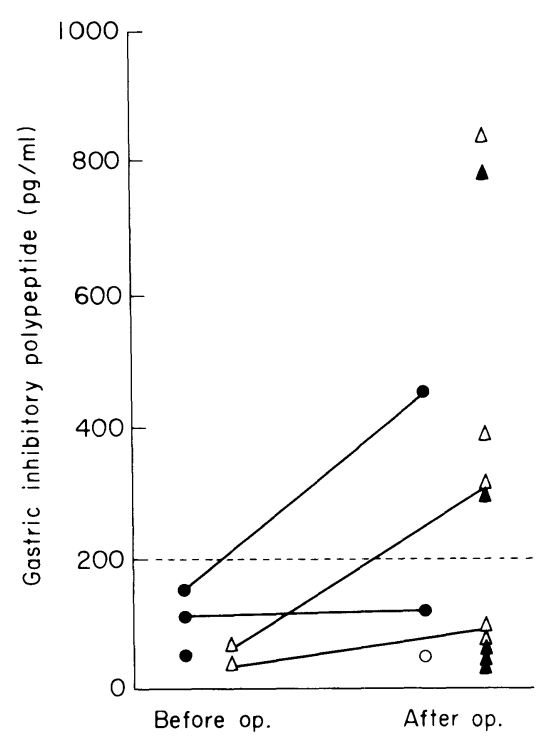

Fig. 2. Serum GIP level in biliary atresia patients. $\bigcirc$, bleeding and jaundice-

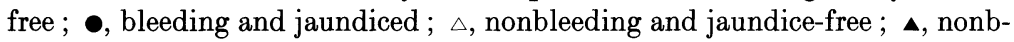
leeding and jaundiced.

\section{Discussion}

Postoperative complications of biliary atresia include peritonitis, ileus, cholangitis and bleeding from esophageal varices due to portal hypertension. Other serious complications include bleeding from the external biliary fistula (Lilly and Stellin 1984; Minifee and Lobe 1988) and progressive hypersplenism (Hayashi et al. 1987). Recently as the number of patients has increased, additional grave complications have been recognized. Though profuse and diffuse bleeding from congested gastric mucosa has been reported in adult patients (Hosking et al. 1987), this condition is rare in childhood.

Possible causes of ulcer formation in biliary atresia include surgical stress, the use of cholagogues and intestinal hormonal imbalance due to surgical biliary diversion (Bismuth and Franco 1978; Nielsen et al. 1980 ; Imamura et al. 1988). The fact that ulcers may be induced by biliary diversion had been reported in animal experiments and clinically, and it is believed that this phenomenon is caused by gastric hypersecretion and an increase in the serum gastrin level (Menguy 1962 ; Bismuth and Franco 1978; Nielsen et al. 1980 ; Imamura et al. 1988). The serum gastrin and GIP levels were increased in this study, possibly due to biliary diversion. However, the formation of gastrointestinal ulcers of erosions was not correlated with increased levels of these hormons in the present study. The existence of jaundice and a history of cholangitis were important risk factors of ulcer formation. Taking these points into account, the incidence of 
ulcer and erosion in biliary atresia is believed to be greater than presently thought. Therefore, periodic routine upper gastrointestinal endoscopy following surgery for biliary atresia should be carried out not only for check of the presence of esophagial varices but for the thorough examination of the gastroduodenal mucosa.

\section{References}

1) Altman, R.P., Chandra, R. \& Lilly, J.R. (1975) Ongoing cirrhosis after successful portoenterostomy in infants with biliary atresia. J. Pediatr. Surg., 10, 685-691.

2) Bismuth, H. \& Franco, D. (1978) Long term results of Roux-en-Y hepaticojejunostomy. Surg. Gynecol. Obstet., 146, 161-167.

3) Hayashi, Y., Ohi, R., Chiba, T., Chiba, T., Mochizuki, I. \& Ishibashi, T. (1987) Effect of partial splenic embolization on hypersplenism in patients with biliary atresia. In: Biliary Atresia, edited by R. Ohi, Professional Postgraduate Serv., Tokyo, pp. 268-271.

4) Hosking, S.W., Kenndy, H.J., Seddon, I. \& Triger, D.R. (1987) The role of propranolol in congestive gastropathy of portal hypertension. Hepatology, 7, 437-441.

5) Imamura, M., Sasaki, I., Funayama, Y., Kamiyama, T. \& Sato, T. (1988) Pathophysiology following biliary reconstruction procedures, with special reference to gastric acid secretion, peptic ulcer, metabolism of carbohydrate and fat, and gastrointestinal hormone release. J. Jpn. Surg. Soc., 89, 1169-1178. (Abstract in English)

6) Lilly, J.R. \& Stellin, G. (1984) Variceal hemorrhage in biliary atresia. J. Pediatr. Surg., 19, 476-479.

7) Menguy, R.B. (1962) Mechanism of gastric hypersecretion in dogs with exclusion of bile or pancreatic juice from the small intestine. Surg. Forum, 13, 300-302.

8) Minifee, P.X. \& Lobe, T.E. (1988) Ostomy closure for recurrent stomal hemorrhage. An indication for prophylactic sclerotherapy in biliary atresia. J. Pediatr. Surg., 23, 630-631.

9) Nielsen, M.L., Jensen, S.L., Malmstrom, J. \& Nielsen, O.V. (1980) Gastrin and gastric acid secretion in hepaticojejunostomy Roux-en-Y. Surg. Gynecol. Obstet., 159, 61-64.

10) Odievre, M. (1980) Late complications after surgery for biliary atresia. In : Cholestasis in Infancy, edited by M. Kasai \& K. Shiraki, University of Tokyo Press, Tokyo, pp. 397-403.

11) Saeki, M., Ogata, T. \& Nakano, M. (1987) Problems in long-term survivors of biliary atresia. In: Biliary Atresia, edited by R. Ohi, Professional Postgraduate Serv., Tokyo, pp. 287-292. 